\title{
Correction to: New asymptotic formulas for sums over zeros of functions from the Selberg class, Ann. Sci. Math. Québec 34 (2010), no 1, 85-108
}

\author{
Kamel Mazhouda ${ }^{1}$ \\ Published online: 8 October 2019 \\ (c) Fondation Carl-Herz and Springer Nature Switzerland AG 2019
}

\section{Correction to: Ann. Math. Québec https://doi.org/10.1007/s40316-019-00124-3}

In this note we make corrections to the above mentioned paper. First, we correct the statement of the main theorem (Theorem 1.1). For this purpose, we correct and add explanation for Lemma 2.1 and its proof. Furthermore, other modifications including the case $v=0$ of Theorem 1.4 were operated. A corrected and revised version of Mazhouda (2010) with all modifications below is appeared recently on arXiv (see, Mazhouda (2019)).

Keywords Selberg class · Sums over zeros · Weil explicit formulas

Mathematics Subject Classification 11M06 $\cdot 11 \mathrm{M} 26 \cdot 11 \mathrm{M} 41$

Résumé Dans cette note, on apporte des corrections au papier mentionné ci-dessus. Tout d'abord, on corrige l'énoncé du théorème principal (Théorème 1.1). Dans ce but, on corrige et on ajoute des explications au Lemme 2.1 et sa preuve. De plus, d'autres modifications incluant le cas $v=0$ du Théorème 1.4 ont été opérées. Une version corrigée et révisée de Mazhouda (2010) avec toutes les modifications ci-dessous est récemment apparue sur arXiv (voir, Mazhouda (2019)).

In this note, we continue to use the notations in [1].

The correct form of Theorem 1.1, i) in [1] should be stated as follows:

i) For $v=u$ or $v=0$, we have

$$
\sum_{\rho} e^{u \rho^{2}-v \rho}=-\frac{d_{F}}{4 \sqrt{\pi u}} \log u+O_{F}\left(u^{-1 / 2}\right), \text { if } u \rightarrow 0^{+},
$$

where $\rho$ runs over all non-trivial zeros of $F(s)$ counted with multiplicity.

The original article can be found online at https://doi.org/10.1007/s40316-019-00124-3.

\footnotetext{
Kamel Mazhouda

kamel.mazhouda@fsm.rnu.tn

1 Department of mathematics, Faculty of Sciences of Monastir, 5000 Monastir, Tunisia
} 
The new statement of Theorem 1.1 is deduced from Lemma 2.1 which should be corrected as follows.

- Statement of Lemma 2.1. Page 92, in the display (4) we made the following changes :

- Replace : $-\left(m_{F}-1\right) e^{u-v}$ by $m_{F} e^{u-v}$.

- Replace : $\frac{e^{\frac{u}{4}-\frac{v}{2 \pi}}}{2 \pi}$ by $\frac{e^{\frac{u}{4}-\frac{v}{2}}}{\pi}$.

- Delete the error term : $O\left(\frac{e^{-u T^{2}}}{T} \log ^{2} T\right)$.

- Replace in the two integral defining $I(\lambda, \mu), e^{-(v+\lambda x)^{2}}$ by $e^{-(v+\lambda x)^{2} / 4 u}$ and $e^{-(v-\lambda x)^{2}}$ by $e^{-(v-\lambda x)^{2} / 4 u}$.

- Proof of Lemma 2.1. There are several points that have to be corrected in the proof of Lemma 2.1.

- We add using reference [8] in [1], the equation

$$
\int_{0}^{+\infty} \frac{1}{\sqrt{4 \pi u}} e^{-(v+\log x)^{2} / 4 u} x^{s} \frac{d x}{x}=e^{u s^{2}-v s},
$$

- We add a detaied proof of the equation (6) (see, [2]).

- We replace in the last line of the display at the top of page $93, \frac{1}{4 \pi}$ by $\frac{1}{2 \pi}$.

- We replace in the display (7) page $93,-\left(m_{F}-1\right) e^{u-v}$ by $m_{F} e^{u-v}, \frac{1}{4 \pi}$ by $\frac{1}{2 \pi}$ and delete the error term $O\left(\frac{e^{-u T^{2}}}{T} \log ^{2} T\right)$.

- We replace in the expression defining $L, \frac{1}{4 \pi}$ by $\frac{1}{2 \pi}$ and in the other expression of $L$ in the bottom of page $93, \frac{1}{4 \pi}$ by $\frac{1}{2 \pi}$ and $\frac{1}{2 \pi}$ by $\frac{1}{\pi}$.

- At the end of the proof of Lemma 2.1, we add another expressions of the two integrals which help us to ends the proof.

We now state the correct form of the case $v=0$ in Theorem 1.4 in [1]. We have

$$
\begin{aligned}
& \sum_{\rho} \int_{-\infty}^{+\infty} f\left(\frac{x}{u}\right) e^{x\left(\rho-\frac{1}{2}\right)} d x=-2 f(0) \log Q \\
& +f\left(0^{-}\right) \sum_{j=1}^{r} \lambda_{j}\left(\log \left[\left(\frac{\lambda_{j}}{2}+\mu_{j}\right) u\right]-\frac{\Gamma^{\prime}}{\Gamma}\left(\frac{\lambda_{j}}{2}+\mu_{j}\right)\right) \\
& +f\left(0^{+}\right) \sum_{j=1}^{r} \lambda_{j}\left(\left(\log \left[\left(\frac{\lambda_{j}}{2}+\overline{\mu_{j}}\right) u\right]-\frac{\Gamma^{\prime}}{\Gamma}\left(\frac{\lambda_{j}}{2}+\overline{\mu_{j}}\right)\right)\right. \\
& +f(0) \sum_{j=1}^{r} \lambda_{j}\left(\frac{\Gamma^{\prime}}{\Gamma}\left(\frac{\lambda_{j}}{2}+\mu_{j}\right)-\frac{\Gamma^{\prime}}{\Gamma}\left(\frac{\lambda_{j}}{2}+\overline{\mu_{j}}\right)\right) \\
& -\sum_{j=1}^{r} \lambda_{j} \int_{0}^{+\infty}\left(f\left(-\lambda_{j} x\right)-f\left(0^{-}\right) e^{-\left(\frac{\lambda_{j}}{2}+\mu_{j}\right) x}\right) \frac{d x}{x} \\
& -\sum_{j=1}^{r} \lambda_{j} \int_{0}^{+\infty}\left(f\left(\lambda_{j} x\right)-f\left(0^{+}\right) e^{-\left(\frac{\lambda_{j}}{2}+\overline{\mu_{j}}\right) x}\right) \frac{d x}{x}+O(u),
\end{aligned}
$$

where $\Lambda_{F}(m)=b(n) \log n$ is the generalized von Mangoldt function. 
Notting that, if $f$ is continuous at 0 , the last formula can be written as follows

$$
\begin{aligned}
\sum_{\rho} \int_{-\infty}^{+\infty} f\left(\frac{x}{u}\right) e^{x\left(\rho-\frac{1}{2}\right)} d x \\
=f(0)\left[-2 \log Q+\sum_{j=1}^{r} \lambda_{j}\left(\log \left|\left(\frac{\lambda_{j}}{2}+\mu_{j}\right) u^{2}\right|\right)\right] \\
\quad-\sum_{j=1}^{r} 2 \lambda_{j} \int_{0}^{+\infty}\left(\frac{f\left(\lambda_{j} x\right)+f\left(-\lambda_{j} x\right)}{2}\right. \\
\left.\quad-f(0) e^{-\left(\frac{\lambda_{j}}{2}+\Re\left(\mu_{j}\right)\right) x} \cos \left(\Im\left(\mu_{j}\right) x\right)\right) \frac{d x}{x}+O(u) .
\end{aligned}
$$

\section{Summary of other changes}

- Page 87, in the ii) of Theorem 1.1, we change the error term $O\left(\frac{e^{-u T^{2}}}{T} \log ^{2} T\right)$ by $O_{F, m}(1)$.

- Page 96, in the display (13), we change in the last equality $\log \left(\frac{\lambda^{2}}{4}\right)$ by $\log \left(\frac{\lambda}{2}\right)$. Then, in the last line of the proof of Lemma 2.2, we change in the equality $: \log \left(\frac{\lambda^{2}}{2}\right)$ by $\log \left(\frac{\lambda}{2}\right)$.

- Page 97 , line-3, we change in the integral in the double-side inequality $e^{-(v+\lambda x)^{2}}$ by $e^{-(v+\lambda x)^{2} / 4 u}$.

- Page 98 and in the end of the proof of Theorem 1.1, an equation which yields the first result of the main theorem (Theorem 1.1) is added :

since $e^{\frac{u}{4}-\frac{v}{2}}=1$ for $u=v$ or $v=0$ as $u \rightarrow 0^{+}$, we obtain

$$
\begin{aligned}
\sum_{\rho} e^{u \rho^{2}-v \rho}= & \frac{d_{F}}{\sqrt{16 \pi u}}\left(\log \frac{1}{u}-\gamma_{0}\right)+\frac{1}{\sqrt{4 \pi u}} \log \left(\frac{q_{F}}{(4 \pi)^{d_{F}}}\right) \\
& +O_{F}(1), \text { if } u \rightarrow 0^{+}, \\
= & -\frac{d_{F}}{4 \sqrt{\pi u}} \log u+O_{F}(1), \text { if } u \rightarrow 0^{+},
\end{aligned}
$$

where $\gamma_{0}$ is the Euler constant. Then, we change in the two equation after the proof the error term $O(1)$ by $O_{F}(1)$.

- Page 99 , in the display (27), we add the factor $\left(\lambda_{j}\right)^{\epsilon / 2}$ to the second and third inequalities.

- Page 102, line 6, we change $f(0)$ by $f\left(0^{-}\right)$and in equation (27), we change in the second integral in the last equality $e^{-x}$ by $e^{-\left(\frac{\lambda}{2}+\mu\right) x}$.

- Page 103, line 4 , in the last integral in the display (24), we change : $e^{-x}$ by $e^{-\left(\frac{\lambda}{2}+\mu\right) x}$ and line 8 (in the last integral), we change $e^{-x}$ by $e^{-\left(\frac{\lambda}{2}+\bar{\mu}\right) x}$. 
- Page 104, the right change of variable is $T=\frac{t}{u \lambda}$, then we get

$$
\begin{aligned}
- & \frac{d_{F}}{2 \pi} \int_{0}^{+\infty} \widehat{f}(-u T) \log \left(\frac{T}{2 \pi}\right) d T \\
= & -\frac{d_{F}}{2 \pi u \lambda} \int_{0}^{+\infty} \widehat{f}\left(-\frac{t}{\lambda}\right) \log \left(\frac{t}{2 \pi u \lambda}\right) d t \\
= & -\frac{d_{F}}{2 \pi u \lambda} \int_{0}^{+\infty} \widehat{f}\left(-\frac{t}{\lambda}\right)(\log t-\log (2 \pi u \lambda)) d t \\
& =\frac{d_{F} \log (2 \pi u \lambda)}{2 u} f(0)-\frac{d_{F}}{2 \pi u \lambda} \int_{0}^{+\infty} \widehat{f}\left(-\frac{t}{\lambda}\right) \log t d t .
\end{aligned}
$$

Hence, in equation (24) we change : $\log (2 \pi u)$ by $\log (2 \pi u \lambda)$.

- Page 106, we change in the statement of Theorem 5.2: $\Re e\left(\lambda_{F}(n)\right)>0$ by $\Re e\left(\lambda_{F}(n)\right) \geq$ 0

Acknowledgements We would like to express our thanks to the anonymous referee for his/her careful reading of the corrected manuscript [2] and the corrigendum, comments and suggestions.

\section{References}

1. Mazhouda, K.: New asymptotic formulas for sums over zeros of functions from the Selberg class. Ann. Sci. Math. Québec 34(1), 85-108 (2010)

2. Mazhouda, K.: New asymptotic formulas for sums over zeros of functions from the Selberg class (2019). arXiv: $1505.01544 \mathrm{v} 2$

Publisher's Note Springer Nature remains neutral with regard to jurisdictional claims in published maps and institutional affiliations. 